Supporting information for

\title{
Molecular Dynamics of a Vanadate-Dipeptide Complex in Aqueous Solution.
}

Michael Bühl

Cartesian coordinates in $\AA$ (BP86/AE1 optimized)

\begin{tabular}{llll}
22 & \multicolumn{4}{l}{} \\
{$\left[\mathrm{VO}(\mathrm{OH})(\mathrm{H} 2 \mathrm{O})\left(\mathrm{glyg} \mathrm{Y}^{\prime}\right)\right]$} & $(1 \mathrm{a})$ & \\
$\mathrm{V}$ & 0.1451453286 & -0.9670126163 & -0.3583909779 \\
$\mathrm{O}$ & 0.7517560126 & -1.6257891963 & -1.9898250533 \\
$\mathrm{O}$ & -1.6685388774 & -0.5602653406 & -0.5761447957 \\
$\mathrm{O}$ & 0.4711030441 & 0.8955913846 & -2.0314048534 \\
$\mathrm{O}$ & -3.5353308765 & 0.4917604381 & 0.1962287397 \\
$\mathrm{O}$ & 0.1502812452 & -2.274974259 & 0.5673882146 \\
$\mathrm{~N}$ & -0.0315491715 & 0.570276533 & 0.905951703 \\
$\mathrm{~N}$ & 2.1857564982 & -0.3362560551 & 0.0572870562 \\
$\mathrm{C}$ & -2.3416107691 & 0.2757297392 & 0.2718268451 \\
$\mathrm{C}$ & -1.3929830329 & 0.8752503696 & 1.3218953144 \\
$\mathrm{C}$ & 0.9532607298 & 1.4845950211 & 1.1757099072 \\
$\mathrm{C}$ & 2.2866340768 & 1.0684151276 & 0.5351319752 \\
$\mathrm{O}$ & 0.8328223899 & 2.5351586002 & 1.8160034301 \\
$\mathrm{H}$ & -1.6427807358 & 0.4104004875 & 2.2977992321 \\
$\mathrm{H}$ & -1.5479480304 & 1.9640631948 & 1.4313113726 \\
$\mathrm{H}$ & 3.1126075387 & 1.2042195782 & 1.2535521508 \\
$\mathrm{H}$ & 2.4659254895 & 1.7197773578 & -0.3350385192 \\
$\mathrm{H}$ & 2.5306546056 & -0.9941765079 & 0.7676574817 \\
$\mathrm{H}$ & 2.7252078069 & -0.493361539 & -0.8026227602 \\
$\mathrm{H}$ & 0.489829828 & 0.1959981768 & -2.7254418224 \\
$\mathrm{H}$ & -0.4262458949 & 1.2849384666 & -2.093179015 \\
$\mathrm{H}$ & 0.8264060152 & -2.6045069469 & -1.9790666513
\end{tabular}

22

[VO $(\mathrm{OH})$ (glygly')].H2O (1b)

$\begin{array}{llll}\mathrm{V} & 0.0882628909 & -0.9906471467 & 0.2441559457 \\ \mathrm{~N} & 0.0057243942 & 0.7315916009 & -0.7471204434 \\ \mathrm{~N} & -1.9337843042 & -0.2724575641 & 0.5148579139 \\ \mathrm{C} & 2.3850311696 & 0.3703426016 & -0.7925538703 \\ \mathrm{C} & 1.2498210617 & 1.2570812834 & -1.3033674226 \\ \mathrm{C} & -1.161022746 & 1.3614865318 & -1.1288168704 \\ \mathrm{C} & -2.3893558702 & 0.6470181941 & -0.5516909454 \\ \mathrm{O} & 0.1053905469 & -1.3323928476 & 2.0539069406 \\ \mathrm{O} & 1.8805278397 & -0.7212835513 & -0.1389256334 \\ \mathrm{O} & -1.4368124581 & 0.7028418726 & 3.124683198 \\ \mathrm{O} & 3.5725899471 & 0.5723905129 & -0.9439103688 \\ \mathrm{O} & -0.3376433172 & -2.2752089206 & -0.6081917338 \\ \mathrm{O} & -1.2366158261 & 2.3533366778 & -1.8578139784 \\ \mathrm{H} & 1.2440695299 & 1.2423862225 & -2.4112465597 \\ \mathrm{H} & 1.4269530414 & 2.3073568934 & -1.0062352742 \\ \mathrm{H} & -2.8680835573 & 0.0662561632 & -1.3589844625 \\ \mathrm{H} & -3.1179073983 & 1.3876607632 & -0.1798278732 \\ \mathrm{H} & -2.5466319595 & -1.0937529001 & 0.5977622519 \\ \mathrm{H} & -1.9100068032 & 0.2077783326 & 1.4508824437 \\ \mathrm{H} & -0.7507630516 & -0.013987878 & 3.0946751982 \\ \mathrm{H} & -0.9170877724 & 1.5324954094 & 3.141906748 \\ \mathrm{H} & 0.0194953029 & -2.2782885044 & 2.3019057411\end{array}$




\begin{tabular}{|c|c|c|c|}
\hline \\
\hline $\mathrm{V}$ & 0.1205159231 & -1.0240580284 & 0.0755843868 \\
\hline $\mathrm{C}$ & -0.8425965667 & -2.0901601046 & -1.1764216206 \\
\hline O & -1.86169259 & -0.5013941263 & 0.4126428174 \\
\hline $\mathrm{O}$ & -3.5008472075 & 1.0507091727 & 0.1882876995 \\
\hline O & 0.1832948497 & -1.7753536863 & 1.5161088789 \\
\hline $\mathrm{N}$ & 0.0826310094 & 1.0221973724 & -0.0916070489 \\
\hline $\mathrm{N}$ & $2.1121458-0$ & $.21663253 \quad 0.80$ & 73035505 \\
\hline C & -2.3124691497 & 0.7086472367 & 0.1414170603 \\
\hline C & -1.2004629156 & 1.6890111843 & -0.2535441249 \\
\hline C & 1.189954154 & $1.8163008451 \quad-$ & 0.1458183402 \\
\hline $\mathrm{C}$ & 2.4764777266 & 1.0080544595 & 0.0887756442 \\
\hline O & 1.2169091836 & 3.0419778183 & -0.3752709961 \\
\hline $\mathrm{H}$ & -1.2611732782 & 2.6089887263 & 0.3600104772 \\
\hline $\mathrm{H}$ & -1.3635677054 & 2.0129060102 & -1.3030144026 \\
\hline $\mathrm{H}$ & 3.221402572 & $1.6359352143 \quad 0$ & .6146414544 \\
\hline $\mathrm{H}$ & 2.8952838364 & 0.7120815177 & -0.888587081 \\
\hline $\mathrm{H}$ & 2.0077204965 & -0.0788504762 & 1.8169831398 \\
\hline $\mathrm{H}$ & 2.7239079269 & -1.0147221405 & 0.6130149434 \\
\hline $\mathrm{H}$ & -1.7721217522 & -1.9749889151 & -0.872921963 \\
\hline O & 1.4942642304 & -1.62751747 & 1.1011137448 \\
\hline $\mathrm{H}$ & 0.977589847 & -2.1051443458 & -1.7872886 \\
\hline \multicolumn{4}{|l|}{21} \\
\hline \multicolumn{4}{|c|}{$\operatorname{mer}-\left[\mathrm{VO}(\mathrm{OH}) 2\left(\mathrm{glygl} \mathrm{y}^{\prime}\right)\right]-\quad(2 \mathrm{c})$} \\
\hline $\mathrm{V}$ & 0.0488760039 & -1.1111189573 & -0.0192695096 \\
\hline O & 0.2289009923 & $-2.70579084 \quad-$ & 0.3120 \\
\hline O & -1.8247200974 & -0.6895589508 & -0.1 \\
\hline O & -3.5053100556 & 0.821387857 & -0.1130 \\
\hline O & 0.1672495001 & -1.1962205916 & 1.8768542167 \\
\hline $\mathrm{N}$ & 0.0631643464 & 1.0421053697 & 0.313 \\
\hline $\mathrm{N}$ & 2.165909854 & -0.5352015245 & 0.2581 \\
\hline $\mathrm{C}$ & -2.3043054159 & 0.5615889586 & -0.0303199667 \\
\hline $\mathrm{C}$ & -1.2399810206 & 1.6596297606 & 0.174 \\
\hline $\mathrm{C}$ & 1.1622923499 & 1.7769182359 & 0.05 \\
\hline $\mathrm{C}$ & .4247120787 & 0.886202603 & 0.09265 \\
\hline $\mathrm{O}$ & .2702196956 & 3.0111914502 & -0.1212837805 \\
\hline $\mathrm{H}$ & -1.543920075 & 2.2643205236 & 1.056 \\
\hline $\mathrm{H}$ & -1.2761608321 & 2.3572343805 & -0.68 \\
\hline $\mathrm{H}$ & 3.2641874099 & 1.3099891915 & 0.492014 \\
\hline $\mathrm{H}$ & 2.7004763658 & $0.922705366 \quad-$ & 1.15924 \\
\hline $\mathrm{H}$ & 2.2853272411 & -0.7242467894 & 1.2610925734 \\
\hline $\mathrm{H}$ & 2.7652706962 & -1.1776549582 & -0.2724781207 \\
\hline $\mathrm{H}$ & 0.0530253252 & -0.2396365096 & 2.0865063633 \\
\hline O & 0.4538308819 & -0.5658377439 & -1.8102149363 \\
\hline $\mathrm{H}$ & 0.4464510998 & -1.4127089409 & -2.3069488997 \\
\hline \multicolumn{4}{|l|}{21} \\
\hline \multicolumn{4}{|c|}{$\left[\mathrm{VO}(\mathrm{OH}) 2\left(\mathrm{glygly} \mathrm{y}^{\prime}\right)\right]-\quad(2 \mathrm{~d})$} \\
\hline $\mathrm{V}$ & 0.8829985141 & -0.7086762092 & -0.2405006031 \\
\hline O & 1.5231527679 & -1.8164152955 & 1.1068283617 \\
\hline 0 & -1.0053851148 & -1.2076023899 & 0.8673641152 \\
\hline O & -3.2707856098 & -1.0014628636 & 0.6607672898 \\
\hline 0 & 2.2326569119 & -0.2139995007 & -0.9863382635 \\
\hline $\mathrm{N}$ & -0.4372286924 & 0.8210842733 & -0.6141992633 \\
\hline $\mathrm{N}$ & 1.3324882894 & 0.9311342177 & 1.2394855467 \\
\hline C & -2.1075496681 & -0.6856497121 & 0.3698968164 \\
\hline C & -1.8289465779 & 0.3997586849 & -0.717620072 \\
\hline C & -0.1978754551 & 2.1031310433 & -0.2161285363 \\
\hline C & 1.1612261266 & 2.2070125432 & 0.5124517554 \\
\hline O & -0.9541257891 & 3.0904268689 & -0.3210393875 \\
\hline $\mathrm{H}$ & -2.5056808652 & 1.2625710682 & -0.5856859972 \\
\hline $\mathrm{H}$ & -2.0345520532 & -0.0508881967 & -1.7092724816 \\
\hline
\end{tabular}




\begin{tabular}{|c|c|c|c|}
\hline $\mathrm{H}$ & 1.1497699736 & 3.1018718933 & 1.1630253133 \\
\hline $\mathrm{H}$ & 1.9935408905 & 2.2882780817 & -0.2088077485 \\
\hline $\mathrm{H}$ & 0.6604280766 & 0.8443493658 & 2.011470564 \\
\hline $\mathrm{H}$ & 2.2794229195 & 0.7777469585 & 1.6030207757 \\
\hline $\mathrm{H}$ & 0.6766857564 & -2.052941677 & 1.5578905502 \\
\hline 0 & 0.1783508482 & -1.8549958095 & -1.49657066 \\
\hline $\mathrm{H}$ & -0.5874364573 & -2.2500875977 & -1.016822487 \\
\hline \multicolumn{4}{|l|}{18} \\
\hline $\mathrm{V}$ & -0.1128748069 & -1.1459581458 & 0.0294100803 \\
\hline O & -0.5480072541 & -1.8440235151 & 1.4516871158 \\
\hline O & 1.7970734966 & -0.7808094448 & 0.1449158014 \\
\hline 0 & 3.5219566012 & 0.6904686012 & 0.032124976 \\
\hline O & -0.2792411372 & -2.1611695689 & $\quad-1.256769322$ \\
\hline $\mathrm{N}$ & -0.0623502268 & 0.9200086576 & -0.1473007482 \\
\hline $\mathrm{N}$ & -2.1774823677 & -0.4901404884 & -0.314233005 \\
\hline $\mathrm{C}$ & 2.3157393011 & 0.4372170188 & 0.0116161302 \\
\hline $\mathrm{C}$ & 1.2536078851 & 1.5365360627 & -0.1781756514 \\
\hline C & -1.1412851509 & 1.7238359581 & 0.0027194346 \\
\hline C & -2.4344025736 & 0.8901718821 & 0.1494560183 \\
\hline $\mathrm{O}$ & -1.1770828752 & 2.9702326231 & 0.0700162287 \\
\hline $\mathrm{H}$ & 1.4513860034 & 2.0584967786 & -1.1377758643 \\
\hline $\mathrm{H}$ & 1.3659809124 & 2.3052252847 & 0.6130674907 \\
\hline $\mathrm{H}$ & -3.2652660424 & 1.3827947469 & -0.3906051065 \\
\hline $\mathrm{H}$ & -2.7021748607 & 0.8487468711 & 1.2203825549 \\
\hline $\mathrm{H}$ & -2.2764016879 & -0.5796514239 & -1.3313122882 \\
\hline $\mathrm{H}$ & -2.7781230198 & -1.1918071316 & 0.1310536477 \\
\hline \multicolumn{4}{|l|}{21} \\
\hline TS & $->4+\mathrm{H} 2 \mathrm{O}$ & $(\mathrm{TS} 2 \mathrm{~b} 4)$ & \\
\hline V & -0.5385335947 & -0.7834957207 & 0.1099884339 \\
\hline $\mathrm{O}$ & -2.6084155022 & -1.4726488622 & 0.092785788 \\
\hline O & -1.6339935901 & 0.8687714844 & 0.1330792858 \\
\hline O & -1.8194102599 & 3.1174901258 & -0.0711597287 \\
\hline O & -0.346920864 & -1.4178650367 & 1.5948986075 \\
\hline $\mathrm{N}$ & 0.8539852874 & 0.7238835786 & -0.116592337 \\
\hline $\mathrm{N}$ & 1.4333751205 & -1.730817321 & -0.3287715873 \\
\hline $\mathrm{C}$ & -1.1360893958 & 2.0934293962 & -0.0264716289 \\
\hline $\mathrm{C}$ & 0.3883269117 & 2.1001996713 & -0.1485867268 \\
\hline $\mathrm{C}$ & 2.2023371527 & 0.5567147996 & -0.0198867042 \\
\hline $\mathrm{C}$ & 2.5869877764 & -0.9282407289 & 0.0922281454 \\
\hline O & 3.0662927652 & 1.4558958386 & 0.0076286775 \\
\hline $\mathrm{H}$ & 0.8266246724 & 2.7008663091 & 0.6743317082 \\
\hline $\mathrm{H}$ & 0.6776424338 & 2.6173953215 & -1.0859379334 \\
\hline $\mathrm{H}$ & 2.8032711117 & -1.1539773448 & 1.1519846695 \\
\hline $\mathrm{H}$ & 3.5071327238 & -1.1230639883 & -0.4918986497 \\
\hline $\mathrm{H}$ & 1.374224783 & -2.6535941552 & 0.1117966241 \\
\hline $\mathrm{H}$ & 1.3367775171 & -1.8430753873 & -1.3443172977 \\
\hline $\mathrm{H}$ & -3.038550373 & -0.6055044609 & -0.0766811098 \\
\hline O & -0.8364283174 & -1.8009626008 & -1.355293186 \\
\hline $\mathrm{H}$ & -1.9307415683 & -1.8081749432 & -0.950678585 \\
\hline
\end{tabular}

